\section{Buchrezension zu: Selbstmedikation für die Kitteltasche}

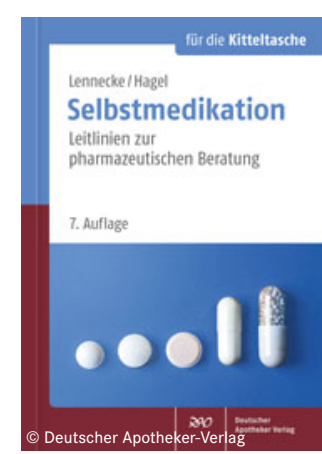

Selbstmedikation für die Kitteltasche

Leilinien zur pharmazeutischen Beratung

Kirsten Lennecke und

Kirsten Hagel

417 S., 7. aktual. und erw. 7. Aufl., Deutscher Apotheker-Verlag, 2021. Flexibel, 24,80 €.

ISBN: 973769275285

Auch als E-Book erhältlich

DOI: $10.1007 / \mathrm{s} 12268-021-1607-3$ (C) Der Autor 2021

Die Arzneitherapie für schwerwiegende Erkrankungen wie $\mathrm{Hy}$ pertonie, Herzinsuffizienz, chronisch-obstruktive Lungenerkrankung, Colitis ulcerosa, peptische Ulkuserkrankung, Glaukom, bösartige Tumorerkrankungen, rheumatoide Arthritis oder Schilddrüsenfunktionsstörungen wird in allen gängigen Pharmakologielehrbüchern ausführlich besprochen. Darüber darf jedoch nicht vergessen werden, dass sich ein sehr großer Bereich der Arzneitherapie im Bereich der Patientenselbstmedikation abspielt und dass die erste (und oft auch einzige) Anlaufstation der Apotheker ist.

Vor diesem Hintergrund ist dieses bereits in der 7. Auflage erscheinende Buch von zwei in der Apotheke sowie in Weiter- und Ausbildung tätigen Autorinnen von Bedeutung. Der geneigte Leser erhält einen umfassenden Einblick in die Welt der „kleinen“ Erkrankungen und Befindlichkeitsstörungen sowie deren Therapie.

Nach einer allgemeinen Einleitung für das Kunden-/Patienten- gespräch werden alphabetisch 109 Themen von Akne über Erkältung, Insektenschutz, Menstruationsbeschwerden, Prellung, Schwindel, Übergewicht bis Zahnungsbeschwerden besprochen. Jedes Thema wird von einem Flussschema zur Beratung eingeleitet - mit klaren Hinweisen, wo ärztliche Konsultation erforderlich wird. Dann werden die einzelnen Arzneistoffe und Arzneimittel besprochen. Es gibt auch allgemeine Hinweise zu jedem Thema. In dem Buch werden nicht nur „schulmedizinische“ Arzneistoffe besprochen, sondern auch eine Vielzahl von pflanzlichen und homöopathischen Arzneistoffen. Der starke Fokus auf Homöopathika hat zwei Gründe: Erstens ist es ein sehr großes Geschäftsfeld für Apotheken. Zweitens wünschen sehr viele Patienten gerade in der Selbstmedikation Homöopathika. Im Gegensatz zu den „schulmedizinischen“ Arzneistoffen haben Homöopathika keinen pharmakologisch definierten Wirkmechanismus, sondern wirken über den Placeboeffekt lindernd. Im Bereich der Selbstmedikation ist dies akzeptabel, solange keine gravierenden Erkrankungen übersehen werden. Denn immer wieder kommt es vor, dass Patienten auch lebensbedrohliche Tumorerkrankungen ausschließlich mit vermeintlich wirksamen und nebenwirkungsarmen Homöopathika behandeln.

Der Preis des Buchs ist angemessen. Das Format (etwas gröBer als DIN A6) und der robuste Kunststoffeinband machen es für die Kitteltasche des Apothekers sehr geeignet. Aber auch für den Nicht-Apotheker, der nicht gleich bei jeder Befindlichkeitsstörung zum Arzt rennen möchte, eignet sich das Buch sehr gut als erste Anlaufstation zum Nachschlagen. Allerdings sollte jeder Leser wissen, dass die Domäne der wissenschaftsgetriebenen evidenzbasierten Medizin häufig verlassen wird.

Roland Seifert,
Medizinische Hochschule

Hannover,

seifert.roland@mh-hannover.de

Diese Rezension erscheint Open Access. ${ }^{\star}$

* Funding note: Open Access funding enabled and organized by Projekt DEAL. Open Access: Dieser Artikel wird unter der Creative Commons Namensnennung 4.0 International Lizenz veröffentlicht, welche die Nutzung, Vervielfältigung, Bearbeitung, Verbreitung und Wiedergabe in jeglichem Medium und Format erlaubt, sofern Sie den/die ursprünglichen Autor(en) und die Quelle ordnungsgemäß nennen, einen Link zu Creative Commons Lizenz beifügen und angeben, ob Änderungen vorgenommen wurden. Die in diesem Artikel enthaltenen Bilder und sonstiges Drittmaterial unterliegen ebenfalls der genannten Creative Commons Lizenz, sofern sich aus der Abbildungslegende nichts anderes ergibt. Sofern das betreffende Material nicht unter der genannten Creative Commons Lizenz steht und die betreffende Handlung nicht nach gesetzlichen Vorschriften erlaubt ist, ist für die oben aufgeführten Weiterverwendungen des Materials die Einwilligung des jeweiligen Rechteinhabers einzuholen. Weitere Details zur Lizenz entnehmen Sie bitte der Lizenzinformation auf http://creativecommons.org/licenses/ by $/ 4.0 /$ deed.de. 